\title{
Exploración de las actitudes y conductas de jóvenes universitarios ante la violencia en las relaciones de pareja
}

\section{Exploration of attitudes and behaviors of college students to violence in intimate relationships}

\author{
Ángel HERNANDO GÓMEZ, Antonio Daniel GARCÍA ROJAS y \\ $\mathrm{M}^{\mathrm{a}}$ del Valle Cecilia MONTILLA CORONADO \\ Universidad de Huelva
}

Recibido: Abril 2012

Aceptado: Julio 2012

\section{Resumen}

En el presente artículo se muestran los resultados de una investigación sobre los conocimientos, actitudes y conductas de los jóvenes ante la violencia en las relaciones de pareja. El alumnado de la Universidad de Huelva ha sido la población objeto de estudio, concretamente de las titulaciones de Educación Social, Psicología y Magisterio (Infantil). Para el estudio se elaboró un instrumento de evaluación y se desarrollaron dos grupos de discusión. Cabe destacar de los datos obtenidos, algunos resultados significativos con respecto al sexo de los participantes y al tener o no pareja en la actualidad. Asimismo, indican la evidencia de la necesidad de informar y formar para la prevención en violencia de género a los/as jóvenes.

Palabras clave: violencia de género, universitarios, conductas, actitudes.

\begin{abstract}
In this paper, we present the results of a study on knowledge, attitudes and behaviors of young people to violence in couple relationships. Students at the University of Huelva has been the target population for the study, specifically those from Social Education, Psychology and Teaching (Kindergarten). For the study developed an evaluation tool and developed two discussion groups. Notably the data obtained some significant results with respect to sex of participants and couple status. They also indicate the evidence of the need for information and training for domestic violence prevention to the young people.
\end{abstract}

Keywords: violence, university, behaviors, attitudes.

Hablar hoy en día de violencia de género significa traer a colación uno de los problemas sociales más acuciantes y de mayor importancia dentro de la prevención e intervención psicológica en los ámbitos familiar, comunitario e individual, pero 
también, por supuesto, dentro del ámbito educativo. En la actualidad, son bastantes los aspectos que se conocen de esta problemática en la edad adulta, sin embargo, esta línea de investigación es todavía muy incipiente en la adolescencia (Fernández Fuertes, Fuertes Martín y Pulido, 2006).

La violencia de género es un problema complejo ya que es multidimensional y está influida por factores relativos al maltratador, a la víctima y a los contextos familiar y sociocultural donde se produce.

Tradicionalmente se ha considerado que existía un perfil de mujer maltratada. Se consideraba que este tipo de actuaciones sólo ocurrían en ambientes desfavorecidos o cuando el agresor presentaba alguna psicopatología. Sin embargo, los estudios concluyen que no se encuentran relaciones consistentes entre las agresiones a mujeres y el estatus socioeconómico o el nivel educativo, la independencia laboral de la mujer (ser o no ama de casa) o algunas características de personalidad de la víctima (pasividad, hostilidad, integración de la personalidad, autoestima, ingesta de alcohol...). Hotaling y Sugarman (1986) concluyen que "el precipitante más influyente para la víctima es ser mujer".

También se ha intentado establecer un perfil del agresor, establecer unas características concretas de personalidad y un estatus socioeconómico y cultural. Sin embargo, lo que encontramos es un grupo heterogéneo. Se ha relacionado con el consumo de alcohol $\mathrm{u}$ otras sustancias, con la experiencia de malos tratos en la infancia o con trastornos de la personalidad. Ninguno de ellos son factores que podamos considerar causales, pues no siempre dan lugar a personas violentas, ni todas las personas que ejercen la violencia de género sufren o han sufrido algunos de estos problemas, aunque sí sea importante tenerlos en cuenta a la hora de establecer estrategias de intervención.

Los factores que inciden en la violencia de género son sociales, estructurales e individuales (de personalidad): los condicionantes de género, la legitimación de la violencia como medio para resolver problemas y otros condicionantes socioculturales que actúan como factores de riesgo. La familia, los iguales y los medios de comunicación se convierten en transmisores de valores que continúan manteniendo ideas claramente diferenciales sobre hombres y mujeres. Será a través de estas identidades como los hombres y las mujeres se construyan a sí mismos. Otro factor relacional importante que se encuentra en muchas víctimas y agresores es la concepción equivocada o tergiversada del ideal de amor. La posesión, los celos, la exclusividad, son ideas relacionadas con la pasión amatoria y llevan a muchas mujeres a sentirse confundidas respecto a lo que les está ocurriendo.

La violencia de género no tiene por qué comenzar después del matrimonio, de hecho, generalmente y cada vez más, ésta se da en el noviazgo o al comienzo de la convivencia (Escoto, González, Muñoz y Salomón, 2007; Gorrotxategi y De Haro, 1999). Encontramos que diversas formas de control exagerado, que han sido pormenorizadamente enumeradas (Ferreira, 1992), comienzan a aparecer de forma 
temprana y, poco a poco, este tipo de conductas abusivas se van haciendo cada vez más frecuentes y extremas.

La violencia durante el noviazgo o también llamada dating violence es definida como todo ataque intencional de tipo sexual, físico o psíquico, de un miembro de la pareja contra el otro en una relación de noviazgo (Health Canada, 1995). Del mismo modo, Close (2005) la define como aquella situación en donde ocurren actos que lastiman a la otra persona, en el contexto de una relación en la que existe atracción y en la que los dos miembros de la pareja se citan para salir juntos.

Esta violencia que se ejerce en las relaciones de noviazgo, relaciones que comienzan por otra parte cada vez a una edad más temprana (Price y Byers, 1999), no es excepcional y se ha encontrado que ésta, en las relaciones de pareja de adolescentes, al igual que la violencia de género en adultos, se extiende en un continuo que va desde el abuso verbal y emocional, hasta la agresión sexual y el asesinato.

En 1998, la Organización Mundial de la Salud (OMS) informó que el 30\% de las estudiantes universitarias habían revelado algún tipo de violencia en sus relaciones de pareja, y con el tiempo, las agresiones verbales se convertían en agresiones físicas. Esta investigación coincide a grandes rasgos con el Servicio de Violencia Familiar de Bilbao que muestra que en el $22 \%$ de los casos registrados, los problemas de violencia empiezan durante el noviazgo (Echeburúa y De Corral, 1998). Datos extraídos de otros países revelan que en el $72 \%$ de los casos atendidos en los últimos años, se detecta que la violencia se inicia también en este periodo de tiempo (Trujano y Mata, 2002).

La revisión de las investigaciones realizadas en los diversos países sobre la violencia en las relaciones de pareja de jóvenes, aporta datos que muestran que la violencia es significativa, e incluso su magnitud es superior a la de las parejas adultas (Jackson, Cram y Seymour, 2000). Específicamente, en el estudio de Kury, ObergfellFuchs y Woessner (2004) las jóvenes determinan que son objeto de más ataques violentos que las mujeres de mayor edad, en concreto entre un $12.5 \%$ y un $28 \%$. Del mismo modo, en un estudio con estudiantes universitarios de 17 naciones: 6 Europeos, 2 del Norte de América, 2 Latino Americanos, 5 Asiáticos, un Australiano y 2 de Nueva Zelanda, los resultados determinan un intervalo entre $15 \%$ a $45 \%$ de jóvenes que agreden a sus parejas en el noviazgo (Straus y Savage, 2005). De forma similar, en una muestra representativa de 863 mujeres universitarias, entre los 18 a los 25 años, el $48 \%$ refirió sufrir violencia y, de éste, el 39\% informó de más de una forma de dicha violencia (Frederick y Susan, 2005).

Es por ello que gran parte del panorama investigador constata que la violencia en el noviazgo es un grave problema que afecta de forma considerable la salud física y mental de los adolescentes y adultos jóvenes de hoy produciéndose con independencia de la edad, raza, orientación sexual, estatus socioeconómico o lugar de residencia (Krug, Dahlberg, Mercy, Zwi y Lozano, 2002; Hernando, 2007; Tjaden y Thoennes, 2000; Makepeace, 1981).

Con respecto a la situación existente en España, una de las investigaciones más reciente es la llevada a cabo por Muñoz-Rivas, Graña, O’Leary y González en 2009 y 
2007. Estos investigadores encontraron una prevalencia de $95.3 \%$ y de $92.8 \%$ de mujeres y de varones, respectivamente, que habían ejercido conductas verbales agresivas, así como una prevalencia de $2 \%$ y 4,6\% de mujeres y de varones, respectivamente, que habían llevado a cabo actos de agresión física. Estos datos confirman que las agresiones verbales son las más comunes y señalan que este tipo de agresiones son realizadas más frecuentemente por las mujeres.

Otra investigación llevada a cabo por González y Santana (2001) que el 7.5\% de los chicos y el $7.1 \%$ de las chicas reconocen que en una o más ocasiones han pegado o empujado a su pareja. Además, según los resultados de otra reciente investigación, un buen número de jóvenes parecen admitir la existencia de agresiones sexuales en sus relaciones de pareja (Fernández Fuertes y Fuertes Martín, 2005). Del mismo modo, existe evidencia que señala que las agresiones de tipo psicológico se presentan antes que las de tipo físico y son más frecuentes que éstas últimas, las físicas $(62 \%$ frente al 46\%) (Cáceres, 2004; Muñoz-Rivas et al., 2007).

Estos datos demuestran que el uso de la violencia no suele surgir de forma espontánea durante el matrimonio o en la vida de pareja; con frecuencia se inicia durante el noviazgo en jóvenes y adolescentes (Serran y Firestone, 2004).

Sensibilizados ante esta realidad, un grupo de profesoras y profesores de la Universidad de Huelva presentamos un Proyecto de Investigación con los siguientes objetivos:

1) Analizar/evaluar los conocimientos, actitudes y conductas del alumnado implicado respecto a la violencia de género.

2) Formar al alumnado participante respecto a la violencia de género. Esta formación se llevará a cabo de forma presencial y de forma virtual.

3) Comprobar la efectividad de un programa de sensibilización/prevención de la violencia de género.

4) Utilizar las Tecnologías de la Información y la Comunicación, de manera específica la plataforma de formación virtual Moodle de la Universidad de Huelva.

5) Detectar posibles casos de violencia en la pareja que se dé entre el alumnado participante.

En el presente artículo presentamos los datos referidos al primer objetivo, es decir, un análisis y una evaluación de los conocimientos, actitudes y conductas con respecto a la violencia de género de una muestra de alumnas y alumnos de la Universidad de Huelva. 


\section{Método}

\section{Participantes}

La muestra del estudio está compuesta por 152 estudiantes de la Universidad de Huelva. Del total de participantes, el $84.9 \%$ eran mujeres $(n=129)$ y el $15.1 \%$ restante eran varones $(n=23)$, con una media de edad de 23 años (rango: 18-47; d.t. 5.8). En cuanto al tipo de estudios que cursaban el 28.9\% estudiaba Psicología (el 7.2\% segundo, el $13.8 \%$ cuarto y el $7.9 \%$ quinto curso), el $46 \%$ Magisterio (23\% la Especialidad de Educación Primaria y el 23\% la de Educación Infantil) y el 25\% Educación Social, distribuyéndose el $8.6 \%$ en primero y el $16.4 \%$ en segundo curso.

Con respecto a los participantes en los Grupos de Discusión, el Grupo 1 estuvo compuesto por 13 estudiantes de Magisterio, 8 chicos de la especialidad de Educación Primaria y 5 chicas de la Educación Infantil. Las edades de estos estudiantes oscilan entre 18 y 28 años, siendo la media de edad 21 años. De entre estos componentes, 3 chicas y 4 chicos dicen tener pareja y 2 chicas y 4 chicos no la tienen. El Grupo 2 estuvo compuesto por 9 estudiantes: 8 cursan $2^{\circ}$ de Educación Social, 4 chicos y cuatro chicas y una chica está en $4^{\circ}$ de Psicología. Las edades se encuentran entre 19 y 42 años, siendo la media de 23 contando al de más edad (1 hombre de 42) y de 19.5 sin él. De entre estos componentes, todos dicen tener pareja menos dos chicas.

\section{Instrumentos}

El cuestionario consta de 70 ítems. En la primera parte del instrumento, se incluyeron datos referidos a la edad, sexo, curso académico, orientación sexual, tipo de noviazgo y duración de la relación de noviazgo. Los instrumentos que se utilizaron para la elaboración del cuestionario fueron seleccionados en función de los objetivos de la investigación (evaluación de los conocimientos, actitudes y conductas con respecto a la violencia de género) así como de la población objeto de estudio (alumnado universitario), siendo los siguientes:

1.-Inventory of Beliefs about Wife Beating (long version) de Saunders, Lynch, Grayson y Linz (1987). Es un cuestionario que está formado por 30 ítems en escala tipo Likert de cinco niveles (desde "totalmente en desacuerdo" hasta "totalmente de acuerdo").

En base a un juicio de expertos se llevó a cabo la selección de los ítems más relevantes de la escala y que mayor correlación tenían con aquellas variables objeto de nuestro estudio. Los 6 ítems que fueron seleccionados fueron: 1, 8, 18, 20, 22 y 29.

2.-Escala de Tácticas de Dominancia y Tácticas Celosas (Dominating and Jealous Tactics Scale; Kasian y Painter, 1992). Es una escala de 22 ítems, 11 de los cuales fueron seleccionados por Kasian y Painter (1992) del Inventario del Maltrato Psicológico de Mujeres (Psychological Maltreatment of Women Inventory) de Tolman $(1989,1999)$, con el objetivo de valorar las diferentes formas de agresión emocional en las relaciones íntimas de jóvenes universitarios. Las alternativas de 
respuesta son cinco comprendidas entre las categorías "nunca" y "muy a menudo" (de 1 a 5 puntos), según la frecuencia con la que se usan las tácticas de dominancia y tácticas celosas, con preguntas bidireccionales: una relativa a la conducta de la persona que responde y la otra referida a la pareja respectiva. De esta forma, se obtienen dos medidas, la que procede de la persona que emite la agresión (agresor/a) y la que resulta de la persona que recibe la agresión (víctima). La escala se compone de dos subescalas que poseen un total de 11 ítems.

Para la construcción del cuestionario final, el grupo de expertos (en función de los criterios previamente establecidos) procedió a la eliminación del ítem 3 y 6 de la escala, quedándose de los 22 posibles, por la subdivisión de cada ítem, en 18.

3.-Inventario de Sexismo Ambivalente (ISA) en adolescentes (De Lemus et al., 2008) basado en la escala original Ambivalent Sexism Inventory (ASI) de Glick y Fiske (1996). Escala tipo likert de 20 items, con seis puntos de respuesta, desde "muy en desacuerdo" hasta "muy de acuerdo" que evalúa el sexismo hostil o tradicional (definido como actitud claramente negativa basada en la supuesta inferioridad o diferencia de las mujeres como grupo) y el sexismo benévolo (definido como conjunto de actitudes interrelacionadas hacia las mujeres que son sexistas en cuanto que las mujeres son vistas de forma estereotipada y limitadas a ciertos roles, pero que tienen un tono afectivo positivo en el perceptor).

4.-Índice de Abuso en la Pareja (Cáceres, 2002), versión española del Index of Spouse Abuse de Hudson y McIntosh (1981). Esta escala está compuesta por 30 ítems que se responden en una escala tipo Likert de cinco puntos: 1 "nunca", 2 "raramente", 3 "ocasionalmente", 4 "con frecuencia" y 5 "casi siempre", la cual permite evaluar la frecuencia de la violencia física y psicológica ejercida por la pareja.

En función de las correlaciones de los ítems de la escala, el grupo de expertos decidió suprimir algunos de los ítems, en concreto, se eliminaron los ítems: 6, 14, 16,26 y 27 , quedando un total de 26 ítems.

\section{Procedimiento}

Las técnicas de análisis de datos empleadas han sido:

- De corte cuantitativo para extraer la información de los cuestionarios, aplicando técnicas de estadística descriptiva y de correlación a través del paquete estadístico SPSS.

- De corte cualitativo (análisis de contenido) para extraer la información. El proceso seguido para el análisis de los grupos de discusión, una vez realizada la transcripción, ha seguido las siguientes fases: reducción de datos, presentación de datos y extracción de conclusiones. 


\section{Resultados}

\section{Análisis cuantitativos: cuestionarios}

Como resultados más significativos nos encontramos con:

$>$ El 63.8\% de los alumnos se muestra muy de acuerdo con que "La Administración Pública" debería de hacer más por ayudar a las mujeres maltratadas.

$>$ El 85.5\% está muy de acuerdo con que las mujeres deben de ser protegidas por la ley.

$>$ El 68.4\% está muy de acuerdo con que si la mujer es golpeada por su marido, debería divorciarse/separarse de él inmediatamente.

$>$ Respecto a "la mejor manera de luchar contra el maltrato es detener a quien lo hizo" hay una mayor dispersión, un $19.7 \%$ se muestra un poco de acuerdo, un $28.3 \%$ y $39.5 \%$ bastante de acuerdo y muy de acuerdo respectivamente.

$>$ El $68.4 \%$ se muestra muy de acuerdo con que si la mujer está siendo atacada por su pareja, debería llamar a la policía.

$>$ Con respecto a si existen diferencias en el grado de sexismo, en función de la variable sexo, nos encontramos que existen diferencias significativas entre hombres y mujeres de la muestra en el sexismo hostil pero no en el benévolo.

$>$ Con respecto a si existen diferencias en el grado de abuso físico y no físico en función del sexo, nos encontramos que no existen. Sin embargo, sí existen diferencias en ambos tipos en función de si los sujetos tienen actualmente pareja o no. Parece ser que se informa de un mayor abuso tanto físico como no físico cuando las personas contestan al cuestionario respecto a su anterior pareja pero sin estar con él/ella actualmente, que cuando se encuentran en un relación, debido a la deseabilidad social.

$>$ Con respecto al "tiempo saliendo juntos" (años) no hay diferencias significativas.

$>$ Hay evidencias significativas de que las mujeres son más dominantes y celosas que los hombres y también son más víctimas de situaciones de dominancia y celosas que los hombres.

$>$ Los que no salen actualmente con alguien son más víctimas de celos y dominancia.

$>$ Los que sí salen actualmente con alguien son menos víctimas de celos.

$>$ En los ítems que se refieren a si ellos son los que agreden no existen diferencias significativas. 


\section{Análisis cualitativos: Grupos de Discusión}

Para el análisis de contenido de los grupos de discusión hemos propuesto un sistema de categorías no excluyentes entre sí que nos permitan explorar los contenidos del discurso de las y los participantes en el grupo en relación con la violencia de género.

El proceso de definición de estas categorías ha constado de dos fases. En un primer momento, se realizó un diseño a priori y, posteriormente, las categorías fueron redefiniéndose en el proceso de codificación de las transcripciones, de manera que su definición fuese más ajustada al criterio de categorización adoptado en acuerdo interjueces por los/as investigadores/as responsables del proceso de categorización, además de generar nuevas categorías que pudiesen reflejar aspectos interesantes no previstos inicialmente.

A continuación describimos cada una de las categorías del análisis de contenido:

1. Caracterización maltrato: Básicamente, pretendemos explorar cómo las y los participantes en los grupos definen el maltrato en cuanto a comportamientos, causas, posibles atribuciones de género, etc.

$>$ Comportamientos: ejemplos de situaciones en las que exista maltrato.

$>$ Causas: motivos que atribuyen al comportamiento del maltratador $\mathrm{o}$ maltratadora.

$>$ Género: opinión sobre si hay prevalencia o no de género en el maltrato, en qué sentido se da, si es que la hay, y si existe alguna diferencia en cuanto al tipo de maltrato en función del mismo.

$>$ Causas de la permanencia: causas que atribuyen a que la persona maltratada permanezca en la relación.

$>$ Modificabilidad de la conducta del maltratador/a: opinión sobre si una persona que maltrata, puede cambiar o no.

2. Pareja Sana: Qué opinan las y los participantes sobre lo que caracteriza a una relación sana. Opiniones de los sujetos sobre qué caracteriza a una relación sana. Entendemos que el mantenimiento de este tipo de relación como una forma de prevención del comienzo de la escalada en la violencia.

3. Opinión personal ante el maltrato: Posiciones, pensamientos, actitudes, juicios morales, etc., del individuo ante el maltrato como fenómeno. Cómo intervenir, propuestas preventivas, estudio de influencias positivas y/o negativas (medios de comunicación...), temáticas más urgentes y formatos más adecuados para este tratamiento.

4. Escalada: Se refiere a la descripción paulatina de maltrato, se presenta un incremento de comportamientos violentos. 
Respecto a la frecuencia de intervenciones que se puede asignar a cada categoría tenemos los siguientes resultados, de más a menos significatividad:

1. Categorización del maltrato: causas, comportamiento, diferencias en cuanto al género, motivos de permanencia en la pareja a pesar del maltrato y por último posibilidad de modificación de conductas en las personas maltratadoras. A continuación recogemos una muestra:

- "La educación que se recibe en casa, cuando el padre llega a casa y exige la comida en la mesa a la madre...".

- "Depende del contexto y de las oportunidades que nos da la vida. Falta de estímulos y de recursos" "el hecho de desarrollarse en un ambiente desfavorable/ altos factores de riesgo...".

- "Los celos, la principal, tener celos a alguien".

- “...posesión...".

- "Estar borracho, suelen refugiarse en el alcohol".

- "La falta de control...".

- "La Iglesia, que ha tratado a la mujer al nivel de la mierda...".

- "Se lleva todo el día fumando porros ahí en la casa y encima, cuando llega la mujer a casa...".

Las causas por las que se mantiene una relación en la que existe maltrato tiene como primer factor el miedo, la inseguridad. Asimismo, los participantes opinan que no tener independencia es lo que ata y hace que se soporte esa situación. Se destaca también la educación recibida que hace ver "normal" esos comportamientos abusivos (sobre todo los individuos que han vivido en su infancia o juventud maltrato en sus padres). Otro motivo es la falta de conciencia de que se está siendo maltratado/a.

Existe un claro posicionamiento hacia la identificación del maltrato físico en los hombres mayoritariamente y psicológico en las mujeres. Es casi unánime la opinión de que el hombre maltrata y la mujer es la maltratada.

El último apartado de esta primera categoría se refiere a la posibilidad o no de modificabilidad en la persona maltratadora. La mayoría de las opiniones va en la línea de que es muy difícil, ya que estas personas ven su actuación como "algo normal", porque no existen castigos fuertes ya que la permanencia en la cárcel se ve escasa en cuanto a tiempo y a mejora de la conducta. El cambio en la conducta de esos individuos se debería trabajar desde la prevención pero cuando se instaura, es muy complicado anularla. Algunos participantes apuntan que para que se recupere, hace falta reconocimiento social y ayuda, la autorreflexión... 
2. Descripción de qué entienden por pareja sana y qué comportamientos se asocian a dicha situación.

Con respecto a la "pareja sana" éstos son los resultados de esta caracterización:

- "Se puede querer a una persona y al mismo tiempo tener tu vida aparte".

- "No hay que ser egoístas hay que dejar salir a la otra persona y confiar en ella".

- "A veces se llega a malentendidos por no ser asertivos".

- "Lo que debe imperar es el afecto entre la pareja".

- "Respeto mutuo".

- "No se puede pretender cambiar a la otra persona, sino aceptarla".

- "Confianza".

- “Comprensión".

- "Cariño".

- "Paciencia".

- "Libertad, apoyarse mutuamente".

- "Tratar a la pareja como un compañero, no como algo tuyo, que sea de tu posesión".

- "Sexo sano".

Con respecto a qué comportamientos se asocian, nombran las siguientes:

- "Coartar las libertades de expresión o de cualquier tipo de la persona que tenemos al lado, no tener su punto de vista en cuenta".

- "Privar de cosas".

- "Falta de respeto, falta de amor a ti mismo y por tanto, a la otra persona".

- "El maltrato puede ser físico o psicológico".

- "Insultos hacia la otra persona".

- "Desprecio, menosprecio, humillación...".

- "Quitar la autoestima".

- "Anularla".

- "Celos insanos que le hacen montar broncas...". 
3. Actitudes y posturas personales ante el maltrato. Son manifiestamente unitarias en cuanto a la condena y al endurecimiento de las penas. También creen mayoritariamente que la intervención más adecuada es la prevención:

- "Hay que trabajar desde abajo, con todos, desde la prevención".

- "La importancia de los valores en la educación. Igualdad, respeto mutuo...".

- "Ante el maltratador hay que intervenir de forma preventiva, para que se detecten cuales son los factores de riesgo, algunas señales, los primeros síntomas...".

- "Es que deberían meterlos en centros específicos que tengan coartada la libertad pero que los estén curando...".

- "Que no salga... toda la vida privado de libertad".

- "Eso no se arregla nunca, eso es algo que va dentro de ti y no cambiará".

- Existe acuerdo así mismo en la influencia de los medios de comunicación y la publicidad, los anuncios "exaltan a los hombres, se les hace superior".

4. Identificación de la escalada como mecanismo de instauración del maltrato. Los participantes reconocen que este hecho no aparece de un día para otro, sin ir poco a poco notando ciertos comportamientos. Casi sin darse cuenta, se van instaurando en la relación de pareja:

- "Si él se porta mal, luego le refuerza con regalos... y al final entra en una dinámica en la que ella misma se autoculpabiliza...".

- "Se rompen las redes sociales... se aísla de los demás...".

- "No sale del círculo vicioso, que puede ser fruto de la escasez de relaciones sociales... porque se ha ido encargando de deteriorar las redes de su pareja...".

Sobre el formato más idóneo para poder formase e intervenir en este campo, nombran: grupos de discusión, plataformas virtuales, role-playing, visionado de películas y debate sobre las mismas, charlas, etc.

\section{Discusión}

A pesar de que en diversas investigaciones se concibe y se mide la violencia desde instancias muy diferentes, los hallazgos de éste y otros estudios indican que la violencia durante el noviazgo es un problema frecuente y merece una mayor atención por parte de las instituciones de educación. Un número importante de jóvenes está implicado en formas de violencia que anuncia la presencia de comportamientos que no son positivos para la relación (control, humillación, manipulación).

En primer lugar, los resultados obtenidos a partir del análisis estadístico y de los grupos de discusión indican la evidencia de la necesidad de informar y formar para la prevención en violencia de género a los/as jóvenes. 
Observamos que el alumnado que ha participado en la experiencia se centra, para hablar de las características del maltrato, en las más usadas en los medios que comunicación, por lo que deducimos que esta información les llega sobre todo a través de estos medios y no por parte de las instituciones ni de la familia, aunque consideran esta última un pilar fundamental en la prevención de la violencia de género.

Un dato a destacar que hemos extraído en este estudio es que se han encontrado evidencias significativas de que las mujeres son más dominantes y celosas que los hombres y también son más víctimas de situaciones de dominancia y celosas que los hombres.

Por otro lado, se ha observado en el alumnado participante que informa de un mayor abuso físico o psíquico si no tienen pareja actualmente. Sin embargo, se informa de un menor abuso si la persona tiene pareja, debido principalmente a la deseabilidad social.

Hemos de mencionar también el claro posicionamiento hacia la identificación del maltrato físico en los hombres y el psicológico en las mujeres. Muchos participantes piensan que es el hombre el que maltrata y la mujer la víctima, lo que nos muestra la falta de concienciación y de formación en la violencia de género de los/as jóvenes.

Las actitudes y posturas que mantienen los participantes ante el maltrato son manifiestamente unitarias en cuanto a la condena y al endurecimiento de las penas. También creen mayoritariamente que la intervención más adecuada es la prevención.

Por último, los resultados confirman la elevada valoración que el alumnado realiza de la formación en torno a la prevención de la violencia de género, centrándose en los grupos de discusión, plataformas virtuales, role-playing, visionado de películas y debate, charlas... como formatos idóneos para su formación. Valoran de forma muy positiva la posibilidad de formarse en la temática y las ventajas que puede traerles su realización a través de los formatos antes mencionados.

\section{Conclusiones}

Ante los resultados obtenidos, se confirma la necesidad de formación de los y las jóvenes universitarios/as en violencia de género en parejas, fundamentalmente a modo preventivo, todo ello a través de actividades que generen reflexión y debate. Esta formación se puede llevar a cabo a través de varios formatos como grupos de discusión, visionado de películas, cursos de libre configuración, etc.

En base a los resultados obtenidos, con este estudio y del interés mostrado por los participantes en formarse en violencia de género en parejas, consideramos que es necesario aplicar un programa de intervención que se ajuste a la problemática, que incremente la capacidad de los/as jóvenes de reconocer y actuar frente al maltrato físico, psíquico y/o sexual, y que se centre en la prevención a través de una metodología activa y participativa, que invite a la reflexión y al análisis crítico. Se trata de formar a los/as jóvenes en la identificación del surgimiento y desarrollo del 
maltrato, formarles en cómo salir de una relación violenta, desechar los mitos del amor y que aprendan las características deseables en una relación de pareja.

Este programa de intervención para la prevención de la violencia de género en parejas jóvenes es sin duda, nuestro objetivo fundamental.

\section{Referencias bibliográficas}

CÁCERES, J. (2004). "Violencia física, psicológica y sexual en el ámbito de la pareja: papel del contexto". Clínica y Salud, 15, 33-54.

CLOSE, S.M. (2005). "Dating violence prevention in middle school and high school youth". Journal of Child and Adolescent Psychiatric Nursing, 18 (1), 2-9.

ECHEBURÚA, E. y DE CORRAL, P. (1998). Manual de violencia familiar. Madrid: Siglo XXI de España Editores.

ESCOTO, Y., GONZÁLEZ, M., MUÑOZ, A. y SALOMÓN, Y. (2007). "Violencia en el noviazgo adolescente". Revista Internacional de Psicología, 8 (2), 1-33.

FERNÁNDEZ FUERTES, A., FUERTES MARTÍN, A. y PULIDO, R. (2006). Evaluación de la violencia en las relaciones de pareja de los adolescentes. Validación del Conflict in Adolescente Dating Relationships Inventory (CADRI)Versión española. International Journal of Clinical and Health Psychology. 6 (2), 339-358.

FERNÁNDEZ FUERTES, A. y FUERTES MARTÍN, A. (2005). "Violencia sexual en las relaciones de pareja de los jóvenes". Sexología Integral, 2, 126-132.

FERREIRA, G.B. (1992). Hombres violentos, mujeres maltratadas: aportes a la investigación y tratamiento de un problema social. Buenos Aires: Editorial Sudamericana.

FREDERICK, A.A. y SUSAN, G. (2005). "Dating violence in college women: Associated Physical injury, Healthcare usage, and Mental health symptoms". Nursing Research, 54(4), 235-242.

GONZÁLEZ, R. y SANTANA, J.D. (2001). Violencia en parejas jóvenes. Análisis y prevención. Madrid: Pirámide.

GONZÁLEZ, R. y SANTANA, J.D. (2001). "La violencia en parejas jóvenes". Psicothema, 13, 127-31.

GORROTXATEGI, M. y DE HARO, I.M. (1999). Materiales Didácticos para la Prevención de la Violencia de Género. Educación Secundaria. Málaga: Consejería de Educación y Ciencia. Junta de Andalucía.

HEALTH CANADA (1995). Dating violence. National Clearinghouse on Family Violence. En http://www.hcsc.gc.ca/hppb/familyviolence/wifeabus.htm. 
HERNANDO, A. (2007). "La prevención de la violencia de género en adolescentes. Una experiencia en el ámbito educativo". Apuntes de Psicología, 26 (3), 325-340.

HOTALING, G.T. y SUGARMAN, D.N. (1986). "An analysis of risk markers in husband to wife violence: the current state of knowledge". Violence and Victims, 1, pp. 101-124.

JACKSON, S.M., CRAM, F. y SEYMOUR, F.W. (2000). "Violence and sexual coercion in high school students' dating relationships". Journal of Family Violence, $15,23-36$.

KURY, H., OBERGFELL-FUCHS, J. y WOESSNER, G. (2004). The extent of family violence in Europe. A comparaison of National Surveys. Violence Agaisnt Women, 10 (7), 749-769.

KRUG, E.G., DAHLBERG, L.L., MERCY, J.A., ZWI, A.B. y LOZANO, R. (Eds.).(2002).World Report on Violence and Health. Geneva: World Health Organization. En Recker, N., Jaycox, L.H., McCaffrey D.F., Ulloa, E., ZanderCotugno, Marshall, G.N. y Shelley, G.A, (2007). Reactions to dating violence among Latino teenagers: An experiment utilizing the Articulated Thoughts in Simulated Situations paradigm. Journal of Adolescence, 30, 893-915.

MAKEPEACE, J.M. (1981). "Courtship violence among collage students". Family Relations, 30, 97-102.

MUÑOZ-RIVAS, M., GRAÑA, J.L., O’LEARY, K.D. y GONZÁLEZ, M.P. (2009). "Prevalence and predictors of sexual aggression in dating relationships of adolescents and young adults". Psicothema, 21 (2), 234-240.

MUÑOZ-RIVAS, M., GRAÑA, J.L., O'LEARY, K.D. y GONZÁLEZ, M.P. (2007). "Aggression in Adolescent Dating Relationships: Prevalence, Justification, and Health Consequences". Journal of Adolescent Health, 40, 298-304.

MUÑOZ-RIVAS, M.J., GRAÑA, J.L., O’LEARY, K.D. y GONZÁLEZ, M.P. (2007). "Physical and psychological aggression in dating relationships in Spanish university students". Psicothema, 19(1), 102-107.

PRICE, E.L. y BYERS, E.S. (1999). The attitudes towards dating violence scales: Development and initial validation. Journal of Family Violence, 14, 351-375. En Recker, N., Jaycox, L. H., McCaffrey D. F., Ulloa, E., Zander-Cotugno, Marshall, G.N., Shelley, G.A, (2007). Reactions to dating violence among Latino teenagers: An experiment utilizing the Articulated Thoughts in Simulated Situations paradigm. Journal of Adolescence, 30, 893-915.

SERRAN, G. y FIRESTONE, P. (2004). "Intimate partner homicida: a review of the male proprietariness and the self-defense theories". Aggression and Violent Behavior, 9, 1-15.

STRAUS, M.A. y SAVAGE, S.A. (2005). "Neglectful behavior by parents in the life history of University students in 17 countries and its relation to violence against 
dating partners". Child Maltreatment: Journal of the American Professional Society on the Abuse of Children, 10(2), 124-135.

TJADEN, P. y THOENNES, N. (2000). Extent, nature, and consequences of intimate partner violence. Findings from the National Violence Against Women Survey. En Recker, N., Jaycox, L.H., McCaffrey D.F., Ulloa, E., Zander-Cotugno, Marshall, G.N. y Shelley, G.A, (2007). Reactions to dating violence among Latino teenagers: An experiment utilizing the Articulated Thoughts in Simulated Situations paradigm. Journal of Adolescence, 30, 893-915.

TRUJANO, P. y MATA, E. (2002). "Relaciones violentas en el noviazgo: un estudio exploratorio". Psicología Conductual, 10(2), 389-408.

\section{Correspondencia con los autores}

Ángel Hernando Gómez

Dirección Postal: Universidad de Huelva. Facultad de Educación. Departamento de Psicología Evolutiva y de la Educación. Campus del Carmen s/n. C.P. 21.007 Huelva. Email: angel.hernando@dpsi.uhu.es

Antonio Daniel García Rojas

Dirección Postal: Universidad de Huelva. Facultad de Educación. Departamento de Educación. Campus del Carmen s/n. C.P. 21.007 Huelva.

Email: antonio.garcia.dedu@uhu.es

$\mathrm{M}^{\mathrm{a}}$ del Valle Cecilia Montilla Coronado

Dirección Postal: Universidad de Huelva. Facultad de Educación. Departamento de Psicología Evolutiva y de la Educación. Campus del Carmen s/n. C.P. 21.007 Huelva. Email: maria.montilla@dpsi.uhu.es 